\title{
Role of correlations in the two-body-marginal problem
}

\author{
Lin Chen, ${ }^{1,2, *}$ Oleg Gittsovich, ${ }^{3,4,5, \dagger}$ K. Modi, ${ }^{6, \ddagger}$ and Marco Piani ${ }^{3,7}, \S$ \\ ${ }^{1}$ Singapore University of Technology and Design, 20 Dover Drive, Singapore 138682 \\ ${ }^{2}$ Institute for Quantum Computing 83 Department of Pure Mathematics, \\ University of Waterloo, 200 University Avenue West, N2L 3G1 Waterloo, Ontario, Canada \\ ${ }^{3}$ Institute for Quantum Computing 8 Department of Physics and Astronomy, \\ University of Waterloo, 200 University Avenue West, N2L 3G1 Waterloo, Ontario, Canada \\ ${ }^{4}$ Institute for Quantum Optics and Quantum Information, \\ Austrian Academy of Sciences, Technikerstr. 21a, A-6020 Innsbruck, Austria \\ ${ }^{5}$ Institute for Theoretical Physics, University of Innsbruck, Technikerstr. 25, A-6020 Innsbruck, Austria \\ ${ }^{6}$ School of Physics, Monash University, Victoria 3800, Australia \\ ${ }^{7}$ Department of Physics and SUPA, University of Strathclyde, Glasgow G4 0NG, UK
}

(Dated: September 20, 2014)

\begin{abstract}
Quantum properties of correlations have a key role in disparate fields of physics, from quantum information processing, to quantum foundations, to strongly correlated systems. We tackle a specific aspect of the fundamental quantum marginal problem: we address the issue of deducing the global properties of correlations of tripartite quantum states based on the knowledge of their bipartite reductions, focusing on relating specific properties of bipartite correlations to global correlation properties. We prove that strictly classical bipartite correlations may still require global entanglement and that unentangled - albeit not strictly classical-reductions may require global genuine multipartite entanglement, rather than simple entanglement. On the other hand, for three qubits, the strict classicality of the bipartite reductions rules out the need for genuine multipartite entanglement. Our work sheds new light on the relation between local and global properties of quantum states, and on the interplay between classical and quantum properties of correlations.
\end{abstract}

\section{INTRODUCTION}

Quantum correlations have a central role in quantum information processing, in quantum foundations, as well as in the physics of strongly correlated systems [1-4]. On one hand, quantum correlations, and in particular entanglement, are a resource that allows one to go beyond what classically possible in many scenarios, from communication tasks, to (measurement-based) quantum computing, to quantum cryptography. On the other hand, the nonclassicality of quantum correlations - be it in the form of non-locality, steering, entanglement, or discord - is one of the most distinctive traits of quantum mechanics, and challenges our understanding of quantum mechanics itself. The interplay between local and global properties of quantum states is a key aspect in the study of quantum correlations, both from a fundamental perspective and an applicative one. For example, we may want to certify the presence of multipartite entanglement in large systems without the - often inaccessible - knowledge of the global state, using instead only the information that comes from reduced states. On the other hand, in condensed-matter physics, because of the typically local - e.g., two-bodyinteractions, relevant properties are dictated by the interplay between the allowed reduced states and global correlations, giving raise to phenomena like frustration [5].

\footnotetext{
*Electronic address: 1inchen0529@gmail.com

${ }^{\dagger}$ Electronic address: oleg.gittsovich@univie.ac.at

$\ddagger$ Electronic address: kavan.modi@monash.edu

$\S$ Electronic address: mpiani@uwaterloo.ca
}

The general study of the relations between the properties of the reduced states and the properties of the global state is known as the quantum marginal problem, which has seen a growing interest in the past years also for the reasons above [6-12].

In this work we study what can be inferred about the quality of the correlations of the global state given information about the two-body reduced states, aiming at answering the question: What correlations need to be present globally to explain what we see locally? In [13] a characterization of multipartite entanglement in terms of even just single-party reduced states (actually, singleparty spectra) was given, but under the assumption of dealing with a pure or quasi-pure global state. In [1416] the possibility of dealing with global mixed states is taken into account, and examples are given where twoqubit separable states are only compatible with global entanglement, intended in the sense of lack of total separability (see Section II for definitions). In [16] examples are also given where genuine multipartite entanglementa much stronger notion of global entanglement - can still be deduced from the properties of the two-body reduced states, but only when these reduced states exhibit bipartite entanglement themselves.

In this work we present several results that complement and generalize those of [14-16]. We offer a brief summary of our findings in Table I. Firstly, we provide examples of triples of bipartite reduced states - in the simplest case, two-qubit states - that, albeit separable, are only compatible with genuine tripartite entanglement (lower-right corner of Table I). As far as we know, this is the strongest "gap" known between the entanglement properties of the 


\begin{tabular}{|c|c|c|c|}
\hline & \multicolumn{2}{|r|}{ Global state } \\
\hline & & Entangled & $\begin{array}{c}\text { Genuinely multipartite } \\
\text { entangled }\end{array}$ \\
\hline $\begin{array}{l}0 \\
.0 \\
.0\end{array}$ & $\begin{array}{c}\text { Fully } \\
\text { classical }\end{array}$ & $\boldsymbol{\nu}$ & $\boldsymbol{x}$ (qubits) \\
\hline 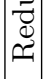 & Separable & $\checkmark[14-16]$ & $\checkmark$ \\
\hline
\end{tabular}

TABLE I: A $\boldsymbol{\checkmark}$ in a cell means that there exist two-body marginal states with the quality of correlations listed in the corresponding row, that are only compatible with global states that have at least the property of correlations listed in the corresponding column. A $\boldsymbol{X}$ means that the inference is not possible: specifically, there are no fully classical twoqubits states that are only compatible with genuine tripartite entanglement (refer to the main text for definitions). Our results correspond to bold symbols, $\boldsymbol{V}$ and $\boldsymbol{X}$. Previous results are reported for completeness and comparison.

marginals and of the global state. Secondly, we address the issue of relating the general quantumness of correlations [2] of reduced states to the quantum correlations of the global state. We find that strictly classical reduced states may still be compatible only with global entanglement (upper-left corner of Table I). Thirdly, we find that, at least for qubits, the strict classicality of the twobody correlations makes it impossible to certify genuine tripartite entanglement based on the knowledge of the reductions: strictly classical two-qubit reduced states are always compatible with a global state that is not genuine tripartite entangled (upper-right corner of Table I).

The rest of the paper is organized as follows. In Section II we define the relevant notions of correlations and classicality, and of compatibility of two-body reduced states in tripartite systems. In Section III we study the relation between the classicality of reductions and their compatibility. In Section IV we prove that unentangled reduced states may only be compatible with genuine multipartite entanglement at the level of the global state. Finally, we conclude in Section V.

\section{CORRELATIONS AND COMPATIBILITY}

We begin by formally defining qualitatively different types of correlations.

Definition 1 Any tripartite mixed state can be written as a mixture of an ensemble of pure states as $\rho_{A B C}=$ $\sum_{i} p_{i}\left|\psi_{i}\right\rangle\left\langle\left.\psi_{i}\right|_{A B C}\right.$. We say that $\rho_{A B C}$ is:

- fully separable, if we can take each $\left|\psi_{i}\right\rangle_{A B C}$ to be fully factorized, e.g. $\left|\alpha_{i}\right\rangle_{A}\left|\beta_{i}\right\rangle_{B}\left|\gamma_{i}\right\rangle_{C}$;

- biseparable, if we can take each $\left|\psi_{i}\right\rangle_{A B C}$ to be unentangled in at least one partition, e.g., $\left|\alpha_{i}\right\rangle_{A}\left|\phi_{i}\right\rangle_{B C},\left|\beta_{i}\right\rangle_{B}|\phi\rangle_{A C}$, or $\left|\gamma_{i}\right\rangle_{C}|\phi\rangle_{A B}$;

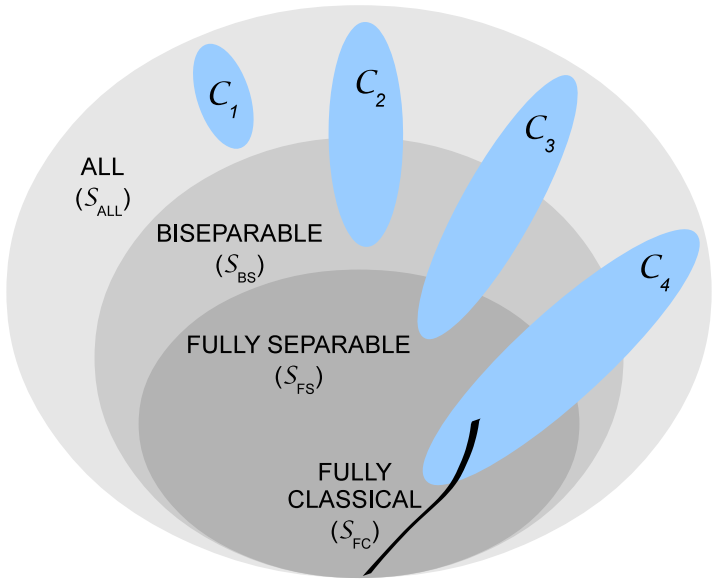

FIG. 1: Hierarchy of correlation classes, and some possible compatibility sets. The set $\mathcal{S}_{\mathrm{FC}}$ is denoted by the line. The set $\mathcal{S}_{\mathrm{GME}}$ of genuinely multipartite states is the complement of $\mathcal{S}_{\mathrm{BS}}$ in the space of all states, $\mathcal{S}_{\mathrm{ALL}}$. The reductions corresponding to the compatibility set $\mathcal{C}_{1}$ are only compatible with genuine multipartite entanglement. The reductions of the states in compatibility set $\mathcal{C}_{2}$ are compatible with entangled biseparable states and genuinely multipartite entangled states, but not separable states. The reductions defining $\mathcal{C}_{3}$ are compatible with a fully separable state as well as with entangled states, but not fully-classical states.

- genuinely multipartite entangled, if for any ensemble there is at least one $\left|\psi_{i}\right\rangle$ with $p_{i}>0$ that is not factorized with respect to any bipartition, i.e., if $\rho_{A B C}$ is not biseparable;

- fully classical, if we can take each $\left|\psi_{i}\right\rangle_{A B C}$ to be of the form $\left|a_{i}\right\rangle_{A}\left|b_{j}\right\rangle_{B}\left|c_{k}\right\rangle_{C}$, with $\left\{\left|a_{i}\right\rangle\right\}, \quad\left\{\left|b_{j}\right\rangle\right\} \quad\left\{\left|c_{k}\right\rangle\right\}$ orthonormal basis on $\mathcal{H}_{A}, \mathcal{H}_{B}$, and $\mathcal{H}_{C}$, respectively, so that, overall, $\rho_{A B C}=\sum_{i j k} p_{i j k}\left|a_{i} b_{j} c_{k}\right\rangle\left\langle a_{i} b_{j} c_{k}\right|$.

Bipartite full classicality and separability are defined similarly: $\rho_{A B}$ is fully classical if $\rho_{A B}=\sum_{i j} p_{i j}\left|a_{i} b_{j}\right\rangle\left\langle a_{i} b_{j}\right|$, for $\left\{\left|a_{i}\right\rangle\right\},\left\{\left|b_{j}\right\rangle\right\}$ orthonormal bases, and separable if $\rho_{A B}=\sum_{i} p_{i}\left|\alpha_{i} \beta_{i}\right\rangle\left\langle\alpha_{i} \beta_{i}\right| . \quad A$ bipartite state is entangled if it is not separable. The notions of full separability and biseparability are redundant for bipartite states.

The set of fully classical states, $\mathcal{S}_{\mathrm{FC}}$, is a subset of the set of fully separable states, $\mathcal{S}_{\mathrm{FS}}$, which in turn is a subset of the set of biseparable states, $\mathcal{S}_{\mathrm{BS}}$; the set of genuinely multipartite entangled states, $\mathcal{S}_{\mathrm{GME}}$ is the is the complement of $\mathcal{S}_{\mathrm{BS}}$ in the space of all states, $\mathcal{S}_{\mathrm{ALL}}$ (see Figure 1). All the mentioned sets apart from $\mathcal{S}_{\mathrm{FC}}$ and $\mathcal{S}_{\mathrm{GME}}$ are convex. A biseparable state may be either separable or entangled in any bipartition, but it is by definition the convex sum of three states that are each separable in one of the bipartitions $A: B C, B: A C$, and $C: A B$. We now move to define formally the notion of compatibility for reduced states. 
Definition 2 Given a triple of three two-party states $\mathcal{E}=\left(\rho_{A B}, \rho_{A C}, \rho_{B C}\right)$, its compatibility set is defined as $\mathcal{C}(\mathcal{E}):=\left\{\sigma_{A B C} \in \mathcal{S}_{A L L} \mid \sigma_{i j}=\rho_{i j}, i j=A B, A C, B C\right\}$. Any compatibility set is a convex set [10], and the property of being part of a given compatibility set defines an equivalence relation. We find it useful to denote by $\mathcal{C}\left(\rho_{A B C}\right)$ the compatibility set associated with the reduced states of $\rho_{A B C}$, i.e., the set of all states that have the same reductions as $\rho_{A B C}$. A triple of two-party states $\mathcal{E}$ is said to be compatible (so that we refer to the triple as triple of reductions) if $\mathcal{C}(\mathcal{E}) \neq \emptyset$, i.e., if there is at least one global state with those reductions.

The following definition links the compatibility of reduced states to the correlation properties of global states.

Definition 3 We say that the reductions $\mathcal{E}$ are incompatible with a set $\mathcal{S}$ (or with the defining correlation property of $\mathcal{S}$ ) if $\mathcal{C}(\mathcal{E}) \cap \mathcal{S}=\emptyset$. We say that (a compatible) $\mathcal{E}$ is only compatible with genuine multipartite entanglement if it is incompatible with $\mathcal{S}_{B S}$.

Figure 1 illustrates the problem of deciding whether certain bipartite reductions necessarily require the presence of global correlations of a certain kind.

We can always expand a generic tripartite state as

$$
\begin{aligned}
\rho_{A B C}= & \rho_{A} \otimes \rho_{B} \otimes \rho_{C}+\chi_{A B C} \\
& +\chi_{A B} \otimes \frac{\mathbb{1}_{C}}{d_{C}}+\chi_{A C} \otimes \frac{\mathbb{1}_{B}}{d_{B}}+\chi_{B C} \otimes \frac{\mathbb{1}_{A}}{d_{A}}
\end{aligned}
$$

where $\rho_{k}$ is the reduced states of party $k$ and $\mathbb{1}_{k} / d_{k}$ is the normalized identity operator on the Hilbert space $\mathcal{H}_{k}$. The bipartite correlation matrices $\chi_{k l}$ can be defined via $\rho_{j k}=\rho_{j} \otimes \rho_{k}+\chi_{j k}$, and satisfy $\operatorname{Tr}_{k}\left[\chi_{k l}\right]=\operatorname{Tr}_{l}\left[\chi_{k l}\right]=0$. It is worth noting that when a bipartite marginal state $\rho_{j k}$ is fully classical, then $\left[\rho_{j} \otimes \rho_{k}, \chi_{j k}\right]=0$, and $\chi_{j k}$ is also diagonal in the same product basis as $\rho_{j k}$. The tripartite correlation matrix $\chi_{A B C}$, which for a fixed $\rho_{A B C}$ can be defined via (1), satisfies $\operatorname{Tr}_{k}\left[\chi_{A B C}\right]=0$, for all $k \in\{A, B, C\}$. For compatible reductions $\mathcal{E}=$ $\left(\rho_{A B}, \rho_{A C}, \rho_{B C}\right)$, the compatibility set $\mathcal{C}(\mathcal{E})$ is spanned by choosing the tripartite correlation matrix $\chi_{A B C}$ so that the resulting operator in (1) is a physical state, i.e., positive semidefinite. On the other hand, to determine whether a triple of bipartite states is compatible, we first check the basic necessary condition that the single party marginals be the same, i.e., $\operatorname{Tr}_{j}\left[\rho_{i j}\right]=\operatorname{Tr}_{k}\left[\rho_{i k}\right]$ for all $\{i, j, k\} \in\{A, B, C\}$. Next, we have to search for a tripartite correlation matrix, $\chi_{A B C}$, such that Eq. (1) is physical. If no such $\chi_{A B C}$ exists, the given states are not compatible.

\section{CLASSICALITY OF REDUCTIONS AND GLOBAL ENTANGLEMENT}

Consider the marginals from the well-known Greenberger-Horne-Zeilinger (GHZ) state $|\mathrm{GHZ}\rangle=$ $(|000\rangle+|111\rangle) / \sqrt{2}: \quad \rho_{A B}=\rho_{B C}=\rho_{A C}=$ $\frac{1}{2}(|00\rangle\langle 00|+| 11\rangle\langle 11|)$. These are fully classical marginals coming from a genuinely tripartite entangled state. However, these marginals are also compatible with $\frac{1}{2}(|000\rangle\langle 000|+| 111\rangle\langle 111|)$, which is fully-classical.

In this section we will first provide an example where fully classical two-body reduced states are not compatible with a global fully classical state, and actually require the presence of entanglement. On the other hand, we will prove that, in the case of three qubits, the fully classical two-body reductions are always compatible with a global states that is not genuine multipartite entangled.

\section{A. Two-body classical states may require global quantumness of correlations}

We will now derive conditions to ensure that some fully-classical marginals cannot be compatible with any global fully-classical state. We start with the following lemma.

Lemma 4 Suppose three states $\rho_{A B}, \rho_{A C}$, and $\rho_{B C}$ are fully classical. Consider the commutators $\Delta_{i j, i k}=$ $\left[\chi_{i j} \otimes \mathbb{1}_{k}, \chi_{i k} \otimes \mathbb{1}_{j}\right]=\left[\rho_{i j} \otimes \mathbb{1}_{k}, \rho_{i k} \otimes \mathbb{1}_{j}\right]$, where the second equality is due to the assumed classicality, i.e., $\left[\rho_{j} \otimes \rho_{k}, \chi_{j k}\right]=0$. Then:

(i) All commutators $\Delta_{i j, i k}$ vanish if and only if there are orthonormal basis $\left\{\left|a_{i}\right\rangle\right\}, \quad\left\{\left|b_{i}\right\rangle\right\}$, $\left\{\left|c_{i}\right\rangle\right\}$, such that $\rho_{A B}=\sum_{i j} p_{i j}\left|a_{i}, b_{j}\right\rangle\left\langle a_{i}, b_{j}\right|$, $\rho_{B C}=\sum_{i j} q_{i j}\left|b_{i}, c_{j}\right\rangle\left\langle b_{i}, c_{j}\right|$, and $\rho_{A C}=$ $\sum_{i j} r_{i j}\left|a_{i}, c_{j}\right\rangle\left\langle a_{i}, c_{j}\right|$.

(ii) If some commutator $\Delta_{i j, i k}$ does not vanish, then:

(a) at least one $\rho_{i}$ is degenerate (i.e., at least two eigenvalues of some $\rho_{i}$ are the same);

(b) there does not exist a tripartite fully classical state that is compatible with $\rho_{A B}, \rho_{B C}$, and $\rho_{A C}$.

Proof. (i) The "if" part is trivial. Let us prove the "only if" part. By hypothesis we may assume

$$
\begin{aligned}
\rho_{A B} & =\sum_{i j} p_{i j}\left|a_{i}, b_{j}\right\rangle\left\langle a_{i}, b_{j}\right| \\
& =\sum_{j} p_{j} \alpha_{j} \otimes\left|b_{j}\right\rangle\left\langle b_{j}\left|=\sum_{i} p_{i}^{\prime}\right| a_{i}\right\rangle\left\langle a_{i}\right| \otimes \beta_{i}, \\
\rho_{A C} & =\sum_{i j} r_{i j}\left|a_{i}^{\prime}, c_{j}\right\rangle\left\langle a_{i}^{\prime}, c_{j}\right| \\
& =\sum_{j} r_{j} \alpha_{j}^{\prime} \otimes\left|c_{j}\right\rangle\left\langle c_{j}\left|=\sum_{i} r_{i}^{\prime}\right| a_{i}^{\prime}\right\rangle\left\langle a_{i}^{\prime}\right| \otimes \gamma_{i}, \\
\rho_{B C} & =\sum_{i j} q_{i j}\left|b_{i}^{\prime}, c_{j}^{\prime}\right\rangle\left\langle b_{i}^{\prime}, c_{j}^{\prime}\right| \\
& =\sum_{j} q_{j} \beta_{j}^{\prime} \otimes\left|c_{j}^{\prime}\right\rangle\left\langle c_{j}^{\prime}\left|=\sum_{i} q_{i}^{\prime}\right| b_{i}^{\prime}\right\rangle\left\langle b_{i}^{\prime}\right| \otimes \gamma_{i}^{\prime},
\end{aligned}
$$


with the orthonormal basis $\left\{\left|a_{i}\right\rangle\right\},\left\{\left|a_{i}^{\prime}\right\rangle\right\}$ on $\mathcal{H}_{A}$, $\left\{\left|b_{i}\right\rangle\right\},\left\{\left|b_{i}^{\prime}\right\rangle\right\}$ on $\mathcal{H}_{B},\left\{\left|c_{i}\right\rangle\right\},\left\{\left|c_{i}^{\prime}\right\rangle\right\}$ on $\mathcal{H}_{C}$, and $\alpha_{j}=$ $\sum_{i} p_{i j}\left|a_{i}\right\rangle\left\langle a_{i}\right| / p_{j}, p_{j}=\sum_{i} p_{i j}$ (similarly for $\beta_{j}$, etc). Then $\Delta_{i j, i k}=0$ implies

$$
\begin{aligned}
& {\left[\chi_{i j} \otimes \mathbb{1}_{k}, \chi_{i k} \otimes \mathbb{1}_{j}\right]} \\
& \quad=\left[\left(\rho_{i j}-\rho_{i} \otimes \rho_{j}\right) \otimes \mathbb{1}_{k},\left(\rho_{i k}-\rho_{i} \otimes \rho_{k}\right) \otimes \mathbb{1}_{j}\right] \\
& \quad=\left[\rho_{i j} \otimes \mathbb{1}_{k}, \rho_{i k} \otimes \mathbb{1}_{j}\right]=0,
\end{aligned}
$$

with $i, j, k \in\{A, B, C\}$. By setting $i=A$ in (5), we have $\left[\alpha_{s}, \alpha_{t}^{\prime}\right]=0, \forall s, t$. Thus the states $\alpha_{s}, \alpha_{t}^{\prime}$ are simultaneously diagonalizable in the orthonormal basis $\left\{\left|a_{i}^{\prime \prime}\right\rangle\right\}$. So we may replace the basis $\left\{\left|a_{i}\right\rangle\right\}$ and $\left\{\left|a_{i}^{\prime}\right\rangle\right\}$ in (2) and (3) by $\left\{\left|a_{i}^{\prime \prime}\right\rangle\right\}$. This replacement may result in the change of $p_{i}^{\prime}, \beta_{i}$ and $r_{i}^{\prime}, \gamma_{i}$. Since there is no confusion, we still use them in (2) and (3).

Next by setting $i=B$ in (5), we have $\left[\beta_{s}, \beta_{t}^{\prime}\right]=0, \forall s, t$. Thus the states $\beta_{s}, \beta_{t}^{\prime}$ are simultaneously diagonalizable in the orthonormal basis $\left\{\left|b_{i}^{\prime \prime}\right\rangle\right\}$. So we may replace the basis $\left\{\left|b_{i}\right\rangle\right\}$ and $\left\{\left|b_{i}^{\prime}\right\rangle\right\}$ in (2) and (4) by $\left\{\left|b_{i}^{\prime \prime}\right\rangle\right\}$. This replacement may result in the change of $q_{i}^{\prime}, \gamma_{i}^{\prime}$. Since there is no confusion, we still use them in (4). Third we set $i=C$ in (5) and repeat the above argument to show that the basis $\left\{\left|c_{i}\right\rangle\right\}$ and $\left\{\left|c_{i}^{\prime}\right\rangle\right\}$ in (3) and (4) can be replaced by the orthonormal basis $\left\{\left|c_{i}^{\prime \prime}\right\rangle\right\}$. So the assertion follows.

(ii) Suppose either condition (a) or (b) is violated. We have that either $\rho_{A}, \rho_{B}$, and $\rho_{C}$ are all non-degenerate respectively in the orthonormal basis $\left\{\left|a_{i}\right\rangle\right\},\left\{\left|b_{i}\right\rangle\right\},\left\{\left|c_{i}\right\rangle\right\}$ (violation of (a)), or that there exists a tripartite fully classical state $\sum_{i, j, k} f_{i j k}\left|a_{i}, b_{j}, c_{k}\right\rangle\left\langle a_{i}, b_{j}, c_{k}\right|$ that is compatible with $\rho_{A B}, \rho_{B C}$, and $\rho_{A C}$ (violation of (b)). In either case we have $\rho_{A B}=\sum_{i j} p_{i j}\left|a_{i}, b_{j}\right\rangle\left\langle a_{i}, b_{j}\right|, \rho_{A C}=$ $\sum_{i j} r_{i j}\left|a_{i}, c_{j}\right\rangle\left\langle a_{i}, c_{j}\right|$ and $\rho_{B C}=\sum_{i j} q_{i j}\left|b_{i}, c_{j}\right\rangle\left\langle b_{i}, c_{j}\right|$. So (i) implies that all commutators $\Delta_{i j, k l}$ vanish, and we have a contradiction.

An immediate consequence of Lemma 4 is the following.

Theorem 5 Let $\rho_{A B}, \rho_{B C}$, and $\rho_{A C}$ be three compatible bipartite fully classical states, such that

(i) they all commute (all commutators $\Delta_{i j, k l}$ of Lemma 4 vanish), or

(ii) all three one-body reduced states $\rho_{A}, \rho_{B}$ and $\rho_{C}$ are non-degenerate.

Then $\rho_{A B}, \rho_{B C}$, and $\rho_{A C}$ are compatible with a fully classical tripartite state.

Proof. The fact that all three single-system reductions are not degenerate implies, by Lemma 4.(iia), that all the commutators $\Delta_{i j, k l}$ defined in Lemma 4 vanish. By Lemma 4.(i) we have that $\rho_{A}, \rho_{B}$, and $\rho_{C}$, are diagonal in the orthonormal bases $\left\{\left|a_{i}\right\rangle\right\},\left\{\left|b_{j}\right\rangle\right\}$, and $\left\{\left|c_{k}\right\rangle\right\}$, respectively, in which $\rho_{A B}, \rho_{B C}$, and $\rho_{A C}$ are explicitly classical. Most importantly, we have

$$
\rho_{X Y}=\sum_{i j}\left|x_{i} y_{j}\right\rangle\left\langle x_{i} y_{j}\left|\rho_{X Y}\right| x_{i} y_{j}\right\rangle\left\langle x_{i} y_{j}\right|,
$$

with $x, y \in\{a, b, c\}$ and $X, Y \in\{A, B, C\}$. Let $\rho_{A B C}$ be any tripartite state with which the three two-body reductions are compatible. Then also the fully classical tripartite state

$$
\sigma_{A B C}=\sum_{i j k}\left|a_{i} b_{j} c_{k}\right\rangle\left\langle a_{i} b_{j} c_{k}\left|\rho_{A B C}\right| a_{i} b_{j} c_{k}\right\rangle\left\langle a_{i} b_{j} c_{k}\right|
$$

has bipartite reduced density matrices $\rho_{A B}, \rho_{B C}$, and $\rho_{A C}$.

Given Theorem 5, in order to construct an example where $\rho_{A B}, \rho_{A C}$, and $\rho_{B C}$ are all fully classical but not compatible with any fully-classical state, we have first of all to construct an example where $\rho_{A B}, \rho_{A C}$, and $\rho_{B C}$ are classical but do not commute with each other. For this, we will need the following lemma. We recall that for a bipartite state $\rho$ acting on the Hilbert space $\mathcal{H}_{A} \otimes \mathcal{H}_{B}$, the partial transpose computed in the standard orthonormal basis $\{|i\rangle\}$ of system $A$, is defined by $\rho^{\Gamma}=\sum_{i j}|j\rangle\langle i| \otimes$ $\langle i|\rho| j\rangle$. One can similarly define the partial transpose $\Gamma_{B}$ on the system $B$.

Lemma 6 Consider three classical-classical two-qubit states

$$
\begin{aligned}
& \rho_{A B}=p(|00\rangle\langle 00|+| 11\rangle\langle 11|)+(1 / 2-p)(|01\rangle\langle 01|+| 10\rangle\langle 10|), \\
& \rho_{B C}=q\left(\left|b_{0}, 0\right\rangle\left\langle b_{0}, 0|+| b_{1}, 1\right\rangle\left\langle b_{1}, 1\right|\right)+(1 / 2-q)\left(\left|b_{0}, 1\right\rangle\left\langle b_{0}, 1|+| b_{1}, 0\right\rangle\left\langle b_{1}, 0\right|\right), \\
& \rho_{A C}=r\left(\left|a_{0}, c_{0}\right\rangle\left\langle a_{0}, c_{0}|+| a_{1}, c_{1}\right\rangle\left\langle a_{1}, c_{1}\right|\right)+(1 / 2-r)\left(\left|a_{0}, c_{1}\right\rangle\left\langle a_{0}, c_{1}|+| a_{1}, c_{0}\right\rangle\left\langle a_{1}, c_{0}\right|\right),
\end{aligned}
$$

where $p, q, r \in(0,1 / 4)$, and any one of $\left\{\left|a_{i}\right\rangle\right\},\left\{\left|b_{i}\right\rangle\right\}$,

$\left\{\left|c_{i}\right\rangle\right\}$ is a real and orthonormal basis in $\mathbf{C}^{2}$. Let

$$
\begin{aligned}
\rho_{A B C}= & -\frac{1}{4} \mathbb{1}_{A} \otimes \mathbb{1}_{B} \otimes \mathbb{1}_{C} \\
& +\rho_{A B} \otimes \frac{\mathbb{1}_{C}}{2}+\rho_{A C} \otimes \frac{\mathbb{1}_{B}}{2}+\rho_{B C} \otimes \frac{\mathbb{1}_{A}}{2} .
\end{aligned}
$$


Then:

(i) If $\rho_{A B C} \geq 0$ then $\rho_{A B C}$ is separable with respect to to the partition $A: B C, B: A C$ and $C: A B$.

(ii) $\rho_{A B}, \rho_{B C}$ and $\rho_{A C}$ are compatible if and only if they are compatible with the biseparable state $\rho_{A B C}$ in (11).

(iii) Suppose $\rho_{A B}, \rho_{B C}$ and $\rho_{A C}$ are compatible. They are compatible with a fully separable state if and only if $\rho_{A B C}$ is fully separable.

Proof. (i) One may directly verify that the state is invariant under partial transposition with respect to any system, i.e., $\rho^{\Gamma_{X}}=\rho$ for $X=A, B, C$. Since $\rho_{A B C} \geq 0$, the assertion follows from [17, Theorem 2].

(ii) The "if" part is trivial; let us prove the "only if" part. Suppose the bipartite marginals $\rho_{A B}, \rho_{B C}$ and $\rho_{A C}$ are compatible with a tripartite state $\rho_{A B C}^{\prime}$. Since $\rho_{A B}$, $\rho_{B C}$, and $\rho_{A C}$ are real, they are also compatible with the real state $\left(\rho_{A B C}^{\prime}+\rho_{A B C}^{\prime *}\right) / 2$, so we can assume that $\rho_{A B C}^{\prime}$ is real without loss of generality. By Eq. (1), there is a Hermitian matrix $\chi_{A B C}$ such that

$$
\rho_{A B C}^{\prime}=\rho_{A B C}+\chi_{A B C} .
$$

Since in our case both $\rho$ and $\rho^{\prime}$ are real, also $\chi$ is real. It follows from Eqs. (8)-(10), and the fact that $\left\{\left|a_{i}\right\rangle\right\}$, $\left\{\left|b_{i}\right\rangle\right\},\left\{\left|c_{i}\right\rangle\right\}$ are real and orthonormal bases, that $\rho_{A B}$, $\rho_{B C}$ and $\rho_{A C}$ are invariant under the local unitary $\sigma_{y} \otimes$ $\sigma_{y}$. So they are compatible with the state

$$
\begin{aligned}
& \frac{1}{2}\left(\rho_{A B C}^{\prime}+\left(\sigma_{y} \otimes \sigma_{y} \otimes \sigma_{y}\right) \rho_{A B C}^{\prime}\left(\sigma_{y} \otimes \sigma_{y} \otimes \sigma_{y}\right)\right) \\
= & \frac{1}{2}\left(\rho_{A B C}+\left(\sigma_{y} \otimes \sigma_{y} \otimes \sigma_{y}\right) \rho_{A B C}\left(\sigma_{y} \otimes \sigma_{y} \otimes \sigma_{y}\right)\right) \\
& +\frac{1}{2}\left(\chi_{A B C}+\left(\sigma_{y} \otimes \sigma_{y} \otimes \sigma_{y}\right) \chi_{A B C}\left(\sigma_{y} \otimes \sigma_{y} \otimes \sigma_{y}\right)\right) \\
= & \rho_{A B C} \\
& +\frac{1}{2}\left(\chi_{A B C}+\left(\sigma_{y} \otimes \sigma_{y} \otimes \sigma_{y}\right) \chi_{A B C}\left(\sigma_{y} \otimes \sigma_{y} \otimes \sigma_{y}\right)\right),
\end{aligned}
$$

where we have used that, from (11),

$$
\left(\sigma_{y} \otimes \sigma_{y} \otimes \sigma_{y}\right) \rho_{A B C}\left(\sigma_{y} \otimes \sigma_{y} \otimes \sigma_{y}\right)=\rho_{A B C} .
$$

We will now argue that, for a real $\chi$,

$$
\chi_{A B C}+\left(\sigma_{y} \otimes \sigma_{y} \otimes \sigma_{y}\right) \chi_{A B C}\left(\sigma_{y} \otimes \sigma_{y} \otimes \sigma_{y}\right)=0,
$$

so that (12) proves that, for a physical state $\rho^{\prime}, \rho$ is also physical, as it corresponds to the convex combination of physical states. The starting point in proving (13) is to observe that every three-qubit correlations matrix $\chi$ is by definition the linear combination of traceless Pauli matrices, i.e.

$$
\chi=\sum_{i, j, k=1}^{3} \chi_{i j k} \sigma_{i} \otimes \sigma_{j} \otimes \sigma_{k} .
$$

Since $\chi$ is Hermitian, all coefficients $\chi_{i j k}$ are real. Moreover, for a real (and hence symmetric) $\chi$ only terms with an even number of $\sigma_{2}=\sigma_{y}$ are present in the expansion, because $\left(\sigma_{2}\right)^{T}=-\sigma_{2}$, while $\sigma_{1}=\sigma_{x}$ and $\sigma_{3}=\sigma_{z}$ are symmetric. On the other hand, $\sigma_{2} \sigma_{m} \sigma_{2}=-\sigma_{m}$ for $m=1,3$, while, obviously, $\sigma_{2} \sigma_{2} \sigma_{2}=\sigma_{2}$. Since each nonzero term in the expansion (14) of $\chi$ contains an even number of $\sigma_{2}$ 's, it will change sign after conjugation by $\sigma_{2} \otimes \sigma_{2} \otimes \sigma_{2}$, i.e.,

$$
\begin{aligned}
& \left(\sigma_{y} \otimes \sigma_{y} \otimes \sigma_{y}\right) \chi_{A B C}\left(\sigma_{y} \otimes \sigma_{y} \otimes \sigma_{y}\right) \\
= & \sum_{i, j, k=1}^{3} \chi_{i j k}\left(\sigma_{y} \otimes \sigma_{y} \otimes \sigma_{y}\right) \sigma_{i} \otimes \sigma_{j} \otimes \sigma_{k}\left(\sigma_{y} \otimes \sigma_{y} \otimes \sigma_{y}\right) \\
= & \sum_{i, j, k=1}^{3} \chi_{i j k}\left(\sigma_{y} \sigma_{i} \sigma_{y}\right) \otimes\left(\sigma_{y} \sigma_{j} \sigma_{y}\right) \otimes\left(\sigma_{y} \sigma_{k} \sigma_{y}\right) \\
= & -\sum_{i, j, k=1}^{3} \chi_{i j k} \sigma_{i} \otimes \sigma_{j} \otimes \sigma_{k} \\
= & -\chi_{A B C} .
\end{aligned}
$$

As argued, this implies $\rho_{A B C} \geq 0$, with biseparability following from (i).

(iii) The "if" part follows from (ii), let us prove the "only if" part. Suppose $\rho_{A B}, \rho_{B C}$ and $\rho_{A C}$ are compatible with a fully separable state $\rho_{A B C}^{\prime}$. From (12), $\rho_{A B C}$ is the convex sum of a few fully separable states. So the assertion follows. This completes the proof.

We are now ready to present our example where $\rho_{A B}$, $\rho_{A C}$, and $\rho_{B C}$ are all fully classical but not compatible with any fully-classical state.

Example 7 Consider the three-qubit state

$$
\begin{aligned}
\rho_{A B C}(q)= & \frac{1}{8}\left(\mathbb{1}_{A} \otimes \mathbb{1}_{B} \otimes \mathbb{1}_{C}+q \mathbb{1}_{A} \otimes \sigma_{1} \otimes \sigma_{1}\right. \\
& \left.+q \sigma_{2} \otimes \mathbb{1}_{B} \otimes \sigma_{2}+q \sigma_{3} \otimes \sigma_{3} \otimes \mathbb{1}_{C}\right),
\end{aligned}
$$

where $\sigma_{i}$, with $i=1,2,3$ are the Pauli matrices and $\frac{-1}{\sqrt{3}} \leq q \leq \frac{1}{\sqrt{3}}$ (for $q$ outside of this interval the matrix $\rho_{A B C}(q)$ is not positive semi-definite), $q \neq 0$. It is not hard to see that each of the bipartite marginals states is fully classical but with respect to different bases. A quick method to verify this assertion is using Theorem 1 in [18]. Moreover the reductions of $\rho_{A B C}(q)$ do not commute with each other. Part (ii) of Lemma 4 implies that there is no tripartite fully classical state that is compatible with these bipartite marginals: $\mathcal{C}\left(\rho_{A B C}(q)\right) \cap \mathcal{S}_{\mathrm{FS}}=\emptyset$. Surprisingly, we can find a value of $q$ for which the fully classical marginals in fact require some global entanglement. Consider the state $\omega_{A B C}=\rho_{A B C}(q=1 / \sqrt{3})$. The range of $\omega_{A B C}$ does not contain any fully factorized pure state, hence it cannot be fully separable. Now, up to a local unitary, the classical bipartite marginals $\omega_{A B}$, $\omega_{A C}, \omega_{B C}$, and the entangled state $\omega_{A B C}$ can be written as (8)-(11), respectively. It follows from Lemma 6.(iii) that $\omega_{A B}, \omega_{A C}$, and $\omega_{B C}$ cannot be compatible with any fully separable state. 
In Appendix A, which focuses on the uniqueness of global states with fixed two-body reductions, we provide also an example of classical reductions compatible with a unique global state that is not fully classical, although fully separable.

\section{B. Classical two-qubit states do not require genuine tripartite entanglement}

Although, as we have just seen, compatible classical marginals may require global quantum correlations or even entanglement, it turns out that for the case of three qubits they will never require the global state to be genuinely multipartite entangled.

To see this, will need an additional lemma.

Lemma 8 Let $\left\{\left|a_{i}\right\rangle\right\},\left\{\left|b_{i}\right\rangle\right\}$ be an orthonormal basis on $\mathcal{H}_{A}$ and $\mathcal{H}_{B}$, respectively. Suppose the bipartite marginals $\rho_{A B}, \rho_{A C}$ and $\rho_{B C}$ are compatible. They are compatible to a non-genuinely entangled tripartite state when one of the following conditions is satisfied: (i) $\rho_{A B}=\sum_{i} p_{i}\left|a_{i}\right\rangle\left\langle a_{i}\right| \otimes \rho_{i}$ and $\rho_{A C}=\sum_{i} q_{j}\left|a_{i}\right\rangle\left\langle a_{i}\right| \otimes \sigma_{i}$. (ii) $\rho_{A B}=\sum_{i} r_{i}\left|a_{i}, b_{i}\right\rangle\left\langle a_{i}, b_{i}\right|$.

Proof. Suppose $\rho_{A B}, \rho_{A C}$ and $\rho_{B C}$ are compatible with $\rho_{A B C}$. If hypothesis (i) is satisfied, then they are also compatible with the state $\sum_{i}\left|a_{i}\right\rangle\left\langle\left. a_{i}\right|_{A} \otimes\right.$ $\mathbb{1}_{B C} \rho_{A B C}\left|a_{i}\right\rangle\left\langle\left. a_{i}\right|_{A} \otimes \mathbb{1}_{B C}\right.$, which is biseparable. On the other hand if hypothesis (ii) is satisfied, then it follows from [19] that there is a quantum channel $\Lambda$ on $\mathcal{H}_{C}$ such that $\rho_{A B C}=\Lambda(|\psi\rangle\langle\psi|)$, for $|\psi\rangle=$ $\sum_{i} \sqrt{r_{i}}\left|a_{i}, b_{i}, i\right\rangle$. So we obtain $\rho_{A C}=\Lambda\left(\sum_{i} r_{i}\left|a_{i}, i\right\rangle\left\langle a_{i}, i\right|\right)$ and $\rho_{B C}=\Lambda\left(\sum_{i} r_{i}\left|b_{i}, i\right\rangle\left\langle b_{i}, i\right|\right)$. Then $\rho_{A B}, \rho_{A C}$ and $\rho_{B C}$ are compatible with the fully separable state $\Lambda\left(\sum_{i} r_{i}\left|a_{i}, b_{i}, i\right\rangle\left\langle a_{i}, b_{i}, i\right|\right)$. This completes the proof.

Now we are ready to give the proof of the following.

Theorem 9 Any three compatible classical-classical twoqubit states $\rho_{A B}, \rho_{B C}$ and $\rho_{A C}$ are compatible with a tripartite biseparable state.

Proof. Suppose some fully classical bipartite marginals $\rho_{A B}, \rho_{B C}$ and $\rho_{A C}$ are only compatible with genuinely entangled states $\rho_{A B C}$. Then the one-party reduced density operators $\rho_{A}, \rho_{B}, \rho_{C}$ have to be the maximally mixed states, $\mathbb{1} / 2$. Indeed, if, without loss of generality in the argument, $\rho_{A}$ is non-degenerate, the two-party reduced states would also be compatible with a global state given by the a locally (on $A$ ) dephased version of $\rho_{A B C}$, which would be separable in $A: B C$, leading to a contradiction. Thus, up to local unitaries, we may assume $\rho_{A B}=p|00\rangle\langle 00|+x| 01\rangle\langle 01|+y| 10\rangle\langle 10|+z| 11\rangle\langle 11|$ where $p+x+y+z=1$ and $0 \leq p \leq 1 / 4$. Since $\rho_{A}=\rho_{B}=\rho_{C}=\mathbb{1} / 2$, we have $p+x=p+y=y+z=1 / 2$. So we obtain $x=y, p=z \in[0,1 / 4]$. Since $\rho_{A B C}$ is genuinely entangled, the cases $p=1 / 4$ and $p=0$ are excluded by Lemma 8.(i) and 8.(ii), respectively. So we obtain $\rho_{A B}$ as Eq. (8). By similar arguments and performing suitable diagonal local unitary gates on systems $A, B$, the classical-classical two-qubit states $\rho_{B C}$ and $\rho_{A C}$ can be simplified to the forms Eqs. (9) and (10), respectively. Meantime, $\rho_{A B}$ is unchanged. Since $\rho_{A B}, \rho_{B C}$ and $\rho_{A C}$ are compatible, it follows from Lemma 6.(ii) that they are compatible with a biseparable state. It gives us a contradiction. So there are no compatible bipartite marginals that are only compatible with genuine entangled states. This completes the proof.

\section{SEPARABLE REDUCTIONS CAN IMPLY GENUINE MULTIPARTITE ENTANGLEMENT}

We have seen that the condition of classicality of marginals is strong enough to exclude the need for global genuine multipartite entanglement. We will now construct non-classical separable marginals that are only compatible with global genuine multipartite entanglement, but first we need to establish some more definitions and notation.

We set $d_{A}=\operatorname{Dim} \mathcal{H}_{A}, d_{B}=\operatorname{Dim} \mathcal{H}_{B}$ and $d_{C}=$ $\operatorname{Dim} \mathcal{H}_{C}$. We denote $r(M), \mathcal{R}(M)$ the rank and range of any square matrix $M$, respectively. A quantum state is a positive semidefinite linear operator $\rho: \mathcal{H} \rightarrow \mathcal{H}$ with $\operatorname{Tr} \rho=1$. We say $\rho_{A B C}$ is a $m \times n \times l$ state by meaning that the reduced density operators satisfy $r\left(\rho_{A}\right)=m$, $r\left(\rho_{B}\right)=n, r\left(\rho_{C}\right)=l$. The ranks of the reduced density operators of $\rho_{A B C}$ are invariant when we perform an invertible local operator (ILO) on $\rho_{A B C}$. That is, let $A=$ $\bigotimes_{i=1}^{3} A_{i} \in \mathrm{GL}:=\mathrm{GL}_{d_{A}}(\mathbf{C}) \times \mathrm{GL}_{d_{B}}(\mathbf{C}) \times \mathrm{GL}_{d_{C}}(\mathbf{C})$ such that $\sigma=A \rho A^{\dagger}$. Then $r\left(\rho_{X}\right)=r\left(\sigma_{X}\right), r\left(\rho_{X Y}\right)=r\left(\sigma_{X Y}\right)$ and $r(\rho)=r(\sigma)$ where $X, Y=A, B, C$. We also denote $\left|a^{*}\right\rangle$ as the vector whose components are the complex conjugate of those of $|a\rangle$. So $|a\rangle$ is real when $|a\rangle=\left|a^{*}\right\rangle$.

Evidently $r\left(\rho^{\Gamma}\right)=r\left(\rho^{\Gamma_{B}}\right)$, where $\Gamma$, we recall, denotes partial transposition. We call the integer pair $\left(r(\rho), r\left(\rho^{\Gamma}\right)\right)$ the birank of $\rho$, and the two integers may be different. For such examples of two-qubit and qubitqutrit separable states, we refer the readers to [20, Table I, II]. Furthermore, we say that $\rho$ is a PPT [NPT] state if $\rho^{\Gamma} \geq 0\left[\rho^{\Gamma}\right.$ has at least one negative eigenvalue]. Evidently, a separable state must be PPT. The converse is true only if $m n \leq 6$ [21, 22].

We say a bipartite state $\rho_{A B}$ is $A$-finite when for any subspace $H \subset \mathcal{H}_{A}$, Dim $H>1$ and any state $|x\rangle \in \mathcal{H}_{B}$, it holds that $H \otimes|x\rangle \not \subset \mathcal{R}\left(\rho_{A B}\right)$. In other words, $\rho_{A B}$ is not $A$-finite when $\mathcal{R}\left(\rho_{A B}\right)$ contains a 2-dimensional subspace spanned by $\left|a_{1}, x\right\rangle,\left|a_{2}, x\right\rangle$ with some linearly independent states $\left|a_{1}\right\rangle,\left|a_{2}\right\rangle$. So if $\rho_{A B}$ is not $A$-finite, there must be infinitely many product states in $\mathcal{R}\left(\rho_{A B}\right)$.

Besides these notions and notation, we will need also the following lemma.

Lemma 10 Suppose the bipartite marginals $\rho_{A B}, \rho_{B C}$ and $\rho_{A C}$ are compatible with $\rho_{A B C}$, and $\rho_{A B}$ is $A$-finite and $B$-finite. Then $\rho_{A B C}$ is either separable with respect 
to the partition $A B: C$, or genuinely multipartite entangled.

Proof. Suppose $\rho_{A B C}$ is biseparable, so $\rho_{A B C}=$ $p \alpha_{A: B C}+q \beta_{B: A C}+(1-p-q) \gamma_{C: A B}$, with $\alpha_{A: B C}$ separable in the $A: B C$ partition (similarly for $\beta_{B: A C}$ and $\gamma_{C: A B}$. We argue that $\alpha_{A: B C}$ is fully separable, and a similar argument will apply to $\beta_{B: A C}$. Let $\alpha_{A: B C}=$ $\sum_{i} p_{i}\left|a_{i}\right\rangle\left\langle\left. a_{i}\right|_{A} \otimes \mid \psi_{i}\right\rangle\left\langle\left.\psi_{i}\right|_{B C}\right.$. Since $\rho_{A B}$ is $B$-finite and $\left|a_{i}\right\rangle \otimes \mathcal{R}\left(\operatorname{Tr}_{C}\left|\psi_{i}\right\rangle\left\langle\psi_{i}\right|\right) \subset \mathcal{R}\left(\rho_{A B}\right)$, any $\left|\psi_{i}\right\rangle$ must be a product state. So $\alpha_{A \text { :BC }}$ is fully separable. Similarly one can show that $\beta_{B: A C}$ is also fully separable, so $\rho_{A B C}$ is separable with respect to $A B: C$.

We are now in the position to prove the following.

Theorem 11 Suppose the triple $\mathcal{E}=\left(\rho_{A B}, \rho_{B C}, \rho_{A C}\right)$ is compatible. Then, $\mathcal{C}(\mathcal{E}) \cap \mathcal{S}_{\mathrm{BS}}=\emptyset$ if all the following conditions are met: (i) for any $i, j \in\{A, B, C\}$, the state $\rho_{i j}$ is $i$-finite and $j$-finite; (ii) $\rho_{B C}$ has birank $\left(r\left(\rho_{B}\right)+\right.$ $\left.1, r\left(\rho_{B}\right)+1\right)$; (iii) $\rho_{A B}$ has birank $(r, s), r \neq s$.

Proof. Suppose $\rho_{A B}, \rho_{B C}$ and $\rho_{A C}$ are compatible with a biseparable state $\rho_{A B C}$. By hypothesis (i) and Lemma 10, we obtain that $\rho_{A B C}$ is separable with respect to the partition $A B: C$. Let $\rho_{A B C}=$ $\sum_{i=0}^{n-1} p_{i} \rho_{i} \otimes\left|c_{i}\right\rangle\left\langle c_{i}\right|$ where $p_{i}>0$, the $\left|c_{i}\right\rangle$ on $\mathcal{H}_{C}$ are pairwise linearly independent. Note that the product subspace $\mathcal{R}\left(\left(\rho_{i}\right)_{B}\right) \otimes\left|c_{i}\right\rangle \subset \mathcal{R}\left(\rho_{B C}\right), \forall i$. This fact and (i) imply that $r\left(\left(\rho_{i}\right)_{B}\right)=1, \forall i$. By similar arguments we have $r\left(\left(\rho_{i}\right)_{A}\right)=1$. We may assume $\rho_{i}=\left|a_{i}, b_{i}\right\rangle\left\langle a_{i}, b_{i}\right|$. Hence $\rho_{A B C}=\sum_{i=0}^{n-1} p_{i}\left|a_{i}, b_{i}, c_{i}\right\rangle\left\langle a_{i}, b_{i}, c_{i}\right|$. By (ii) we have $n \geq d+1$ where $d=r\left(\rho_{B}\right)$. Without loss of generality, we may assume that the states $\left|b_{i}\right\rangle, i=0, \cdots, d-1$ span $\mathcal{R}\left(\rho_{B}\right)$. We choose a suitable ILO $V$ such that $V\left|b_{i}\right\rangle \propto|i\rangle, i=0, \cdots, d-1, V\left|b_{i}\right\rangle \propto\left|f_{i}\right\rangle, i \geq d$ and $\left|f_{d}\right\rangle$ is real. By performing $V$ on the state $\rho_{A B C}$, we have

$$
\begin{aligned}
\sigma_{A B C} & =(I \otimes V \otimes I) \rho_{A B C}(I \otimes V \otimes I)^{\dagger} \\
& =\sum_{i=0}^{d-1} q_{i}\left|a_{i}, i, c_{i}\right\rangle\left\langle a_{i}, i, c_{i}\left|+\sum_{i=d}^{n-1} q_{i}\right| a_{i}, f_{i}, c_{i}\right\rangle\left\langle a_{i}, f_{i}, c_{i}\right|,
\end{aligned}
$$

and $q_{i}>0$ for any $i$. Since the operation $V$ does not change the rank of quantum states, it follows from (ii) that $\sigma_{B C}$ has birank $(d+1, d+1)$. Recall that the $\left|c_{i}\right\rangle$ are pairwise linearly independent. Since (i) is not changed under ILOs, it follows from (i) that $\left|f_{d}\right\rangle$ is not parallel to any state $|i\rangle$-otherwise $\rho_{B C}$ would not be $B$-finite. Since $\left|f_{d}\right\rangle$ is real, the two $(d+1)$-dimensional subspaces $\mathcal{R}\left(\sigma_{B C}\right)$ and $\mathcal{R}\left(\sigma_{B C}^{\Gamma}\right)$ are equal and spanned by $\left|i, c_{i}\right\rangle, i=$ $0, \cdots, d-1$ and $\left|f_{d}, c_{d}\right\rangle$. So the states $\left|f_{i}, c_{i}\right\rangle,\left|f_{i}^{*}, c_{i}\right\rangle \in$ $\mathcal{R}\left(\sigma_{B C}\right)$, for any $i>d$. Then (i) implies that these $\left|f_{i}\right\rangle$ are real up to an overall phase. So $\sigma_{A B}=\sigma_{A B}^{\Gamma_{B}}$. It implies $r\left(\rho_{A B}\right)=r\left(\rho_{A B}^{\Gamma}\right)$ which is a contradiction with (iii). Therefore $\rho_{A B}, \rho_{B C}$ and $\rho_{A C}$ are not compatible to any non-genuinely entangled state. This completes the proof.

We will now make use of Theorem 11 and offer an example of separable marginals only compatible with genuinely entangled tripartite states.
Example 12 Consider the family of rank- $(d+1)$ states on $\mathbb{C}^{d+1} \otimes \mathbb{C}^{d+1} \otimes \mathbb{C}^{d+1}$ given by $\rho_{A B C}=p_{1} \sigma_{A B C}+$ $\sum_{m=2}^{d} p_{m}|m m m\rangle\langle m m m|$, with

$$
\sigma_{A B C}=\frac{2}{3}|\xi\rangle\left\langle\xi\left|+\frac{1}{3}\right| 111\right\rangle\langle 111|,
$$

$|\xi\rangle=\frac{1}{2}|010\rangle+\frac{1}{2}|100\rangle+\frac{1}{\sqrt{2}}|001\rangle, p_{1}>0$, and $p_{m} \geq 0$. It is easy to see that the only biseparable pure state in $\mathcal{R}\left(\sigma_{A B C}\right)$ is $|111\rangle$. The bipartite reduced density operators of $\sigma_{A B C}$ are

$$
\begin{aligned}
\sigma_{A B} & =\frac{1}{3}\left|\Phi^{+}\right\rangle\left\langle\Phi^{+}\left|+\frac{1}{3}\right| 00\right\rangle\left\langle 00\left|+\frac{1}{3}\right| 11\right\rangle\langle 11|, \\
\sigma_{B C} & =\sigma_{A C}=\frac{1}{2}|\zeta\rangle\left\langle\zeta\left|+\frac{1}{6}\right| 00\right\rangle\left\langle 00\left|+\frac{1}{3}\right| 11\right\rangle\langle 11|,
\end{aligned}
$$

with $\left|\Phi^{+}\right\rangle=\frac{1}{\sqrt{2}}(|01\rangle+|10\rangle)$ and $|\zeta\rangle=\sqrt{\frac{2}{3}}|01\rangle+\sqrt{\frac{1}{3}}|10\rangle$.

The three two-qubit marginals $\sigma_{A B}, \sigma_{B C}$ and $\sigma_{A C}$ are positive under partial transposition (PPT), so they are separable [22]. Hence, $\rho_{A B}, \rho_{B C}$ and $\rho_{A C}$ are separable too; they also evidently satisfy condition (i) of Theorem 11. Furthermore, $\rho_{B C}$ has birank $(d+2, d+2)$, while $r\left(\rho_{B}\right)=d+1$, and $\rho_{A B}$ has birank $(d+2, d+3)$. So also conditions (ii) and (iii) of Theorem 11 are satisfied, and we conclude that $\rho_{A B}, \rho_{B C}$ and $\rho_{A C}$ are only compatible with genuinely tripartite entangled states.

The example shows that for any fixed local dimension $d$, there exist triples of two-qudit separable states that are only compatible with genuine multipartite entanglement. The "core" of our construction is the genuine multipartite entangled three-qubit state $\sigma_{A B C}$ of Eq. (16). It turns out that $\sigma_{A B C}$ is actually the only state compatible with its reductions. The proof of this is given in the Appendix. It is worth comparing this with the results of [23]. There it was proven that for almost all pure entangled states of three qubits $|\eta\rangle$ it holds $\mathcal{C}(|\eta\rangle\langle\eta|)=\{|\eta\rangle\langle\eta|\}$, with the exception of states of the generalized GHZ form $|\mathrm{gGHZ}\rangle=$ $\sqrt{p}|000\rangle+\sqrt{1-p}|111\rangle$ (up to local unitary transformations), which satisfy, e.g., $\{|\mathrm{gGHZ}\rangle\langle\mathrm{gGHZ}|, p| 000\rangle\langle 000|+$ $(1-p)|111\rangle\langle 111|\} \subset \mathcal{C}(|g G H Z\rangle\langle g G H Z|)$. Interestingly, the only three-qubit pure states that have separable reduction are of the generalized GHZ form [24]. This implies that any three-qubit state $\rho$ such that (i) its reductions are separable and (ii) $\mathcal{C}(\rho) \cap \mathcal{S}_{\mathrm{BS}}=\emptyset$, must be mixed. Since the state $\sigma_{A B C}$ has rank two, we can think of it as the simplest possible example that satisfies (i) and (ii), with the additional property of being uniquely determined by its reductions. We generalize Example 12 in several ways, all presented in the Appendix.

\section{A. Genuine multipartite entanglement from separable reductions is a robust feature}

While we showed that there exist genuine multipartite states whose compatibility set contains only genuine multipartite states, it is natural to ask how common this 
phenomenon is, i.e., whether such states have finite volume in the set of all states. This is important also from the point of view of the potential realization of such states in the lab, which can never be perfect. We answer this question in the affirmative.

We introduce a parameter of compatibility of a tripartite state $\rho_{A B C}$ with $\mathcal{E}=\left(\sigma_{A B}, \sigma_{B C}, \sigma_{A C}\right)$ as $D\left(\rho_{A B C} \mid \mathcal{E}\right):=\left\|\rho_{A B}-\sigma_{A B}\right\|_{2}^{2}+\left\|\rho_{B C}-\sigma_{B C}\right\|_{2}^{2}+$ $\left\|\rho_{A C}-\sigma_{A C}\right\|_{2}^{2}$, where we have used the Hilbert-Schmidt norm $\|X\|_{2}=\sqrt{\operatorname{Tr}\left(X^{\dagger} X\right)}[29]$. We further define $D_{\mathrm{BS}}(\mathcal{E}):=\min _{\rho \in \mathcal{S}_{\mathrm{BS}}} D\left(\rho_{A B C} \mid \mathcal{E}\right)$. We have $D_{\mathrm{BS}}(\mathcal{E})>0$ for any triple $\mathcal{E}$ such that $\mathcal{C}(\mathcal{E}) \cap \mathcal{S}_{\mathrm{BS}}=\emptyset$, even if the triple of reduced states is compatible, as in Example 12. Finally, given a tripartite state $\sigma_{A B C}$, we define $D\left(\rho_{A B C} \mid \sigma_{A B C}\right):=D\left(\rho_{A B C} \mid\left(\sigma_{A B}, \sigma_{B C}, \sigma_{A C}\right)\right)$ and $D_{\mathrm{BS}}\left(\sigma_{A B C}\right):=\min _{\rho \in \mathcal{S}_{\mathrm{BS}}} D\left(\rho_{A B C} \mid \sigma_{A B C}\right)$.

Now, consider a genuinely entangled multipartite state $\bar{\sigma}_{A B C}$ with separable reductions such that $D_{\mathrm{BS}}\left(\bar{\sigma}_{A B C}\right)>$ 0 , and the convex combination of $\bar{\sigma}_{A B C}$ with an arbitrary fully separable state $\rho^{\mathrm{FS}}: \tau_{p}\left(\bar{\sigma}_{A B C}, \rho^{\mathrm{FS}}\right):=(1-$ $p) \bar{\sigma}_{A B C}+p \rho^{\mathrm{FS}}$, for $0 \leq p \leq 1$. Since the set of biseparable states is closed, there exists $\bar{p}>0$ such that $\tau_{p}\left(\bar{\sigma}_{A B C}, \rho^{\mathrm{FS}}\right)$ is genuine multipartite entangled for all $\rho^{\mathrm{FS}}$ and all $0 \leq p<\bar{p}$. Since $\rho^{\mathrm{FS}}$ is fully separable, so are the two-party reduced states of $\tau_{p}\left(\bar{\sigma}_{A B C}, \rho^{\mathrm{FS}}\right)$. Furthermore, since $D$ is continuous, there exists $\bar{p}_{D}>0$ such that $D_{\mathrm{BS}}\left(\tau_{p}\left(\bar{\sigma}_{A B C}, \rho^{\mathrm{FS}}\right)\right)>0$ for all $\rho^{\mathrm{FS}}$ and all $0 \leq p<\bar{p}_{D}$.

For any local finite dimensions, the set of fully separable states has non-zero volume among all states, because there exists a ball of fully separable states around the maximally mixed state [25]. Thus, the argument above proves that also the set of tripartite states whose twoparty marginals are separable but only compatible with genuine multipartite entanglement has non-zero volume.

\section{CONCLUSIONS}

We analyzed the relation between the character of correlations of tripartite states and the ones exhibited by their bipartite reductions, i.e., a version of the quantum marginal problem that focuses on the compatibility of bipartite reductions with certain global properties. We constructed examples where separable reductions are only compatible with genuine multipartite entanglement. Up to our knowledge, this is the "largest" known separation between the character of correlations of bipartite reductions and what can be inferred about the quality of correlations of the global state, based only on the knowledge of the reductions. On the other hand, at least for qubits we were able to prove that compatible reductions that are fully classical can always originate from a biseparable global state. Nonetheless, bipartite reductions that are fully classical may still require the presence of some entanglement in the global state. Our results show that the relation between global and "local" correlations is far from trivial. Notably, the notion of fully classical correlations is strong enough to "break" the need for genuine multipartite entanglement, but not the potential need for global entanglement altogether. An interesting open question is whether compatible classical-classical marginals in high dimension are always compatible with a biseparable tripartite state. Another question is how to quantitatively bound the certifiable genuine multipartite entanglement in terms of the non-classicality of the twobody reductions, at least in the three-qubit case. The latter problem is reminiscent of the case of entanglement distribution, where the non-classicality of correlationsrather than the entanglement- - present between a quantum carrier and distant labs constitutes a bound on the entanglement that can be generated between the labs by exchanging the carrier $[26,27]$.

\section{Acknowledgments}

We are grateful to A. Acìn and O. Gühne for useful discussions. LC started this work when at the Institute for Quantum Computing \& Department of Pure Mathematics, University of Waterloo, and was mainly supported by MITACS and NSERC; LC's research was funded in part by the Singapore National Research Foundation under NRF Award NRF-NRFF2013-01. OG is grateful for the support of the Austrian Science Fund (FWF) and Marie Curie Actions (Erwin Schrödinger Stipendium J3312-N27). KM was supported by the John Templeton Foundation, National Research Foundation and the Ministry of Education (Singapore) during the completion of this work. MP acknowledges support from NSERC, CIFAR, and Ontario Centres of Excellence.

\section{Appendix A: On the uniqueness of global states compatible with given reductions}

We first prove that $\sigma_{A B C}$ in Eq. (16) in the paper is the only state compatible with its reductions, a fact of interest in its own.

Proposition 13 For $\sigma_{A B C}$ in Eq. (16) it holds $\mathcal{C}\left(\sigma_{A B C}\right)=\left\{\sigma_{A B C}\right\}$.

Proof. Suppose $\rho=\rho_{A B C}$ has the same reductions as $\sigma_{A B C}$, i.e., $\rho \in \mathcal{C}\left(\sigma_{A B C}\right)$. We can always write its spectral decomposition as $\rho=\sum_{i=0}^{7} p_{i}\left|\psi_{i}\right\rangle\left\langle\psi_{i}\right|$, where $\left|\psi_{i}\right\rangle=$ $\sqrt{q_{i}}\left|0, \alpha_{i}\right\rangle+\sqrt{1-q_{i}}\left|1, \varphi_{i}\right\rangle$, and $\left|\alpha_{i}\right\rangle,\left|\varphi_{i}\right\rangle \in \mathcal{H}_{B} \otimes \mathcal{H}_{C}, \forall i$. We have $\sigma_{B C}=\sum_{i} p_{i}\left(q_{i}\left|\alpha_{i}\right\rangle\left\langle\alpha_{i}\left|+\left(1-q_{i}\right)\right| \varphi_{i}\right\rangle\left\langle\varphi_{i}\right|\right)$. It follows from Eq. (5) in the paper that $r\left(\sigma_{B C}\right)=3$. So any four states of $\left|\alpha_{i}\right\rangle, i=0, \cdots, 7$ are linearly dependent. Using the freedom in the choice of the pure-state ensemble representation of a mixed states [28], we can choose a suitable linear combination of $\left|\psi_{i}\right\rangle, i=0,1,2,3$, such that it is equal to $|1\rangle_{A}\left|\varphi_{3}^{\prime}\right\rangle_{B C}$. So the state can be written as $\rho=\sum_{i=0}^{3} r_{i}\left|\psi_{i}^{\prime}\right\rangle\left\langle\psi_{i}^{\prime}\left|+\sum_{i=4}^{7} p_{i}\right| \psi_{i}\right\rangle\left\langle\psi_{i}\right|$ where $\left|\psi_{3}^{\prime}\right\rangle=|1\rangle_{A}\left|\varphi_{3}^{\prime}\right\rangle_{B C}$. By applying this procedure to an- 
other four states $\left|\psi_{0}^{\prime}\right\rangle,\left|\psi_{1}^{\prime}\right\rangle,\left|\psi_{2}^{\prime}\right\rangle,\left|\psi_{j}\right\rangle$ with $j=4,5,6,7$ respectively, we can realize $\left|\psi_{j}\right\rangle=|1\rangle_{A}\left|\varphi_{j}^{\prime}\right\rangle_{B C}$.

By relabeling the states, we can write $\rho=$ $\sum_{i=0}^{2} p_{i}^{\prime}\left|\psi_{i}\right\rangle\left\langle\psi_{i}\left|+p_{3}^{\prime}\right| 1\right\rangle\langle 1| \otimes \rho_{0}$ with $\rho_{0}$ on $\mathcal{H}_{B} \otimes \mathcal{H}_{C}$. We have $\mathcal{R}\left(|1\rangle\langle 1| \otimes\left(\rho_{0}\right)_{B}\right) \subset \mathcal{R}\left(\sigma_{A B}\right)$ and $|11\rangle \in \mathcal{R}\left(\sigma_{A B}\right)$ by Eq. (17). Since $\sigma_{A B}$ is X-finite for $X=A, B$, we have $\left(\rho_{0}\right)_{B}=|1\rangle\langle 1|$. By the similar argument we can show $\left(\rho_{0}\right)_{C}=|1\rangle\langle 1|$. So we have $\rho=\sum_{i=0}^{2} p_{i}^{\prime}\left|\psi_{i}\right\rangle\left\langle\psi_{i}\right|+$ $p_{3}^{\prime}|111\rangle\langle 111|$.

Let $\left|\psi_{i}\right\rangle=\sum_{j, k, l=0}^{1} c_{i, m}|j k l\rangle$ where $i=0,1,2$ and $m=$ $4 j+2 k+l$. By Eq. (17) we have $c_{i 2}=c_{i 4}, c_{i 3}=c_{i 5}$. By Eq. (18), we have $c_{i 2}=c_{i 1} / \sqrt{2}$ and $c_{i 6}=c_{i 5} / \sqrt{2}$. These equations imply for $i=0,1,2$, we have

$$
\left|\psi_{i}\right\rangle=c_{i 0}|000\rangle+c_{i 1}\left(|001\rangle+\frac{1}{\sqrt{2}}|010\rangle+\frac{1}{\sqrt{2}}|100\rangle\right)+c_{i 3}\left(|011\rangle+|101\rangle+\frac{1}{\sqrt{2}}|110\rangle\right)+c_{i 7}|111\rangle .
$$

The coefficients of $|11\rangle\langle 11|$ in both $\sigma_{A B}$ and $\sigma_{A C}$ are $1 / 3$, so $c_{03}=c_{13}=c_{23}=0$. By replacing $\left|\psi_{i}\right\rangle, i=0,1,2$ by a suitable linear combination of them, we may assume $c_{11}=c_{21}=c_{20}=0$. So the tripartite state can be rewritten as $\rho=\sum_{i=0}^{2} p_{i}^{\prime \prime}\left|\psi_{i}\right\rangle\left\langle\psi_{i}\right|$, where

$$
\left|\psi_{0}\right\rangle=c_{00}^{\prime}|000\rangle+c_{01}^{\prime}\left(|001\rangle+\frac{1}{\sqrt{2}}|010\rangle+\frac{1}{\sqrt{2}}|100\rangle\right)+c_{07}^{\prime}|111\rangle, \quad\left|\psi_{1}\right\rangle=c_{10}^{\prime}|000\rangle+c_{17}^{\prime}|111\rangle, \quad\left|\psi_{2}\right\rangle=|111\rangle .
$$

Since $r\left(\sigma_{B C}\right)=3$, we have $c_{01}^{\prime} \neq 0$. By Eq. (17), we have $c_{01}^{\prime} c_{00}^{\prime}=c_{01}^{\prime} c_{07}^{\prime}=0$. So $c_{00}^{\prime}=c_{07}^{\prime}=0$. By Eq. $(17)$ again, we have $p_{0}^{\prime \prime}=\frac{2}{3},\left|c_{01}^{\prime}\right|=\frac{1}{\sqrt{2}}$, and $c_{10}^{\prime}=0$. Now we see $\rho=\sigma_{A B C}$ in Eq. (16). This completes the proof.

We further derive (and later use in Appendix B) the following lemma.

Lemma 14 Suppose $\rho_{A B}, \rho_{B C}, \rho_{A C}$ are only compatible with a tripartite state $\rho_{A B C}$, and $\sigma_{A B}, \sigma_{B C}, \sigma_{A C}$ are compatible with another tripartite state $\sigma_{A B C}$. If $\mathcal{R}\left(\sigma_{A B C}\right) \subseteq \mathcal{R}\left(\rho_{A B C}\right)$, then $\sigma_{A B C}$ is the only state with which $\sigma_{A B}, \sigma_{B C}, \sigma_{A C}$ are compatible.

Proof. Suppose $\sigma_{A B}, \sigma_{B C}, \sigma_{A C}$ are compatible with another state $\sigma_{A B C}^{\prime} \neq \sigma_{A B C}$. Since $\mathcal{R}\left(\sigma_{A B C}\right) \subseteq \mathcal{R}\left(\rho_{A B C}\right)$, we may find a small enough $p>0$ and a tripartite state $\alpha_{A B C}$, such that $\rho_{A B C}=p \sigma_{A B C}+(1-p) \alpha_{A B C}$. So the bipartite reductions $\rho_{A B}, \rho_{B C}, \rho_{A C}$ are compatible with the state $p \sigma_{A B C}^{\prime}+(1-p) \alpha_{A B C}$, which is different from $\rho_{A B C}$. It gives us a contradiction.

We conclude this section presenting separable marginals that are only compatible with a unique quantum correlated (unentangled) state.

Proposition 15 The separable states $\rho_{A B}=\rho_{B C}=$ $\rho_{A C}=p|00\rangle\langle 00|+(1-p)| a, a\rangle\langle a, a|$, where $|a\rangle=\frac{1}{\sqrt{2}}(|0\rangle+$ $|1\rangle)$, are only compatible with the separable state $\rho_{A B C}=$ $p|000\rangle\langle 000|+(1-p)| a, a, a\rangle\langle a, a, a|$.

Proof. We will use the following observation in the proof and it is easy to verify. For any $X, Y \in\{A, B, C\}$, there are only two product states $|00\rangle,|a, a\rangle \in \mathcal{R}\left(\rho_{X Y}\right)$, they also span the space $\mathcal{R}\left(\rho_{X Y}\right)$. That is, any state in $\mathcal{R}\left(\rho_{X Y}\right)$ is the linear combination of $|00\rangle$ and $|a, a\rangle$.

It is clear that $\rho_{A B}, \rho_{B C}, \rho_{A C}$ are compatible with $\rho_{A B C}$. Suppose they are compatible with another three- qubit state $\sigma_{A B C}=\sum_{i} p_{i}\left|\psi_{i}\right\rangle\left\langle\psi_{i}\right|$. By applying the observation to system $B, C$ we have $\left|\psi_{i}\right\rangle=f_{i}\left|\alpha_{i}, 00\right\rangle+$ $g_{i}\left|\beta_{i}, a, a\right\rangle$ with some complex numbers $f_{i}, g_{i}$. By applying the observation to system $A, B$ we have $g_{i}\left|\beta_{i}\right\rangle \propto|a\rangle$, and hence $f_{i}\left|\alpha_{i}\right\rangle \propto|0\rangle$. So we may assume $\left|\psi_{i}\right\rangle=$ $f_{i}^{\prime}|000\rangle+g_{i}^{\prime}|a, a, a\rangle$. As a result, the range of the state $\sigma_{A B C}$ is spanned by the product states $|000\rangle,|a, a, a\rangle$. By simple algebra one can see that the only feasible $\sigma_{A B C}$ compatible to $\rho_{A B}, \rho_{B C}$ and $\rho_{A C}$ is the convex sum of $|000\rangle\langle 000|$ and $|a, a, a\rangle\langle a, a, a|$. By using the condition $\rho_{X Y}=\sigma_{X Y}$ we obtain $\sigma_{A B C}=\rho_{A B C}$. This completes the proof.

\section{Appendix B: Generalizations of Example 12}

We provide here some further examples of states with separable reductions that are only compatible with genuine multipartite entanglement, also making use of Proposition 13 and Lemma 14.

Note that $|111\rangle \in \mathcal{R}\left(\sigma_{A B C}\right)$ for $\sigma_{A B C}$ in Eq. (16). It follows from Lemma 14 that for any $p \in(0,1)$, the separable states $p \sigma_{A B}+(1-p)|11\rangle\left\langle 11\left|, p \sigma_{A C}+(1-p)\right| 11\right\rangle\langle 11|$, and $p \sigma_{B C}+(1-p)|11\rangle\langle 11|$ are uniquely compatible with the state $p \sigma_{A B C}+(1-p)|111\rangle\langle 111|$. So we have generated a family of separable bipartite marginals which are uniquely compatible with a genuinely entangled state, extending Example 12.

We now generalize Example 12 to a different family of states that satisfy the conditions in Theorem 11. Let $\sigma_{A B C}$ be as in Eq. (16), and the product state $|a, b\rangle \in \mathcal{R}\left(\sigma_{B C}\right) \cap \mathcal{R}\left(\sigma_{B C}^{\Gamma}\right)$. Such product state always exists because $r\left(\sigma_{B C}\right)=r\left(\sigma_{B C}^{\Gamma}\right)=3$, and there is a product state in any 2-dimensional two-qubit subspace. For example, we can choose $|a, b\rangle=\left(\frac{1}{\sqrt{2}}, \frac{1}{\sqrt{2}}\right) \otimes\left(\sqrt{\frac{1}{3}}, \sqrt{\frac{2}{3}}\right)$. 
We have the following corollary now.

Corollary 16 Let $\vec{p}=\left(p_{1}, \cdots, p_{n}\right), \sum_{i=1}^{n} p_{i}=1, p_{1}>$ $0, p_{i} \geq 0$. For $i>1$ suppose the product states $\left|a_{i}, b_{i}\right\rangle \in$ $\mathcal{R}\left(\sigma_{B C}\right) \cap \mathcal{R}\left(\sigma_{B C}^{\Gamma}\right)$ where $\left|a_{i}\right\rangle$ is real and $\sigma_{B C}$ is the reduced density operator of the state $\sigma_{A B C}$ in Eq. (16). The three reduced density operators of the three-qubit state $\sigma_{\vec{p}}=p_{1} \sigma_{A B C}+\sum_{i=2}^{n} p_{i}\left|a_{i}, a_{i}, b_{i}\right\rangle\left\langle a_{i}, a_{i}, b_{i}\right|$ are only compatible with genuinely entangled states.

Proof. It is sufficient to show that the three reduced density operators $\left(\sigma_{\vec{p}}\right)_{A B},\left(\sigma_{\vec{p}}\right)_{A C}$, and $\left(\sigma_{\vec{p}}\right)_{B C}$ satisfy the three conditions (i), (ii), and (iii) in Theorem 11. Recall that $\sigma_{A B C}$ satisfies these conditions. Since $\left|a_{i}, a_{i}\right\rangle \in$ $\mathcal{R}\left(\sigma_{A B}\right)$ and $\left|a_{i}, b_{i}\right\rangle \in \mathcal{R}\left(\sigma_{B C}\right)=\mathcal{R}\left(\sigma_{A C}\right)$, we have $\mathcal{R}\left(\left(\sigma_{\vec{p}}\right)_{X Y}\right)=\mathcal{R}\left(\sigma_{X Y}\right)$ for any $X, Y \in\{A, B, C\}$. So condition (i) is satisfied. Next, the same argument shows that $\left(\sigma_{\vec{p}}\right)_{A B}$ has birank $(3,4)$, which is exactly condition (iii). Third, the hypothesis $\left|a_{i}, b_{i}\right\rangle \in \mathcal{R}\left(\sigma_{B C}\right) \cap \mathcal{R}\left(\sigma_{B C}^{\Gamma}\right)$ and $\left|a_{i}\right\rangle$ is real imply that the birank of $\left(\sigma_{\vec{p}}\right)_{B C}$ is $(3,3)$. So condition (ii) is also satisfied.
[1] R. Horodecki, P. Horodecki, M. Horodecki, K. Horodecki, Rev. Mod. Phys. Phys. 81, 865-942 (2009).

[2] K. Modi, A. Brodutch, H. Cable, T. Paterek, V. Vedral, Rev. Mod. Phys. 84, 1655-1707 (2012).

[3] N. Brunner, D. Cavalcanti, S. Pironio, V. Scarani, S. Wehner, arXiv:1303.2849 (2013).

[4] J. Eisert, M. Cramer, M.B. Plenio, Rev. Mod. Phys. 82, 277 (2010).

[5] K. Binder and A. P. Young, Rev. Mod. Phys. 58, 801 (1986); H. T. Diep (ed.), Frustrated spin systems (World Scientific, Singapore, 2005); C. M. Dawson and M. A. Nielsen, Phys. Rev. A 69, 052316 (2004)

[6] S. B. Bravyi, Quantum Inf. and Comp., 4, 012 (2004).

[7] A. Klyachko, arXiv:quant-ph/0409113 (2004).

[8] M. Christandl, G. Mitchison, Commun. Math. Phys., 3, 261 (2006).

[9] M. Christandl, M. B. Sahinoglu, M. Walter, arXiv:1210.0463 (2012).

[10] J. Chen, Z. Ji, D. Kribs, Z. Wei, B. Zeng, J. Math. Phys. 53, 102201 (2012).

[11] Peter D. Johnson and Lorenza Viola, Phys. Rev. A 88, 032323 (2013).

[12] J. Chen, Z. Ji, D. Kribs, N. Lutkenhaus, B. Zeng, arXiv:1310.3530 (2013).

[13] M. Walter, B. Doran, D. Gross, and M. Christandl, Science, 340, 1205-1208 (2013)

[14] G. Toth, C. Knapp, O. Gühne, and H. J. Briegel, Phys. Rev. Lett. 99, 250405 (2007).

[15] G. Toth, C. Knapp, O. Gühne, and H. J. Briegel, Phys. Rev. A 79, 042334 (2009).
[16] L. E. Wurflinger, J.-D. Bancal, A. Acin, N. Gisin, and T. Vertesi, Phys. Rev. A 86, 032117 (2012)

[17] B. Kraus, J. I. Cirac, S. Karnas, and M. Lewenstein, Phys. Rev. A 61, 062302 (2000).

[18] L. Chen, E. Chitambar, K. Modi, G. Vacanti, Phys. Rev. A 83, 020101(R) (2011).

[19] M. Christandl and A. Winter, J. Math. Phys. 45, 829 (2004).

[20] L. Chen and D.Ž. Doković, Phys. Rev. A 86, 062332 (2012).

[21] A. Peres, Phys. Rev. Lett. 77 (1996), 1413-1415.

[22] M. Horodecki, P. Horodecki, and R. Horodecki, Phys. Lett. A 223, 1 (1996).

[23] N. Linden, S. Popescu, and W. K. Wootters, Phys. Rev. Lett. 89, 207901

[24] A. V. Thapliyal, Phys. Rev. A 59, 3336 (1999)

[25] L. Gurvits, H. Barnum, Phys. Rev. A 68, 042312 (2003)

[26] T. K. Chuan, J. Maillard, K. Modi, T. Paterek, M. Paternostro, and M. Piani, Phys. Rev. Lett. 109, 070501 (2012)

[27] A. Streltsov, H. Kampermann, and D. Bruss, Phys. Rev. Lett. 108, 250501 (2012)

[28] L. P. Hughston, R. Josza, and W. K. Wootters, Phys. Lett. A 183, 14 (1993).

[29] We make this choice for the sake of concreteness, but our argument is only based on continuity of $D$ in its arguments and the fact that $D\left(\rho_{A B C} \mid \mathcal{E}\right)$ is positive and vanishes if an only if $\rho_{A B C} \in \mathcal{C}(\mathcal{E})$. 